\title{
Self-Tuning Blind Identification and Equalization of IIR Channels
}

\author{
Miloje Radenkovic \\ Department of Electrical Engineering, College of Engineering and Applied Science, University of Colorado at Denver, \\ Denver, CO 80127-3364, USA \\ Email:mradenko@carbon.cudenver.edu
}

Tamal Bose

Department of Electrical and Computer Engineering, Center for High-speed Information Processing (CHIP), Utah State University, Logan, UT 84322, USA

Email: tbose@ece.usu.edu

\section{Zhurun Zhang}

Department of Electrical and Computer Engineering, Center for High-speed Information Processing (CHIP), Utah State University, Logan, UT 84322, USA

Email: zhurunz@microsoft.com

Received 10 September 2002 and in revised form 18 February 2003

\begin{abstract}
This paper considers self-tuning blind identification and equalization of fractionally spaced IIR channels. One recursive estimator is used to generate parameter estimates of the numerators of IIR systems, while the other estimates denominator of IIR channel. Equalizer parameters are calculated by solving Bezout type equation. It is shown that the numerator parameter estimates converge (a.s.) toward a scalar multiple of the true coefficients, while the second algorithm provides consistent denominator estimates. It is proved that the equalizer output converges (a.s.) to a scalar version of the actual symbol sequence.
\end{abstract}

Keywords and phrases: blind identification, self-tuning equalization, recursive estimation, digital filtering, parameter convergence.

\section{INTRODUCTION}

Intersymbol interference (ISI) imposes limits on data transmission rates in many physical channels. Traditionally, channel equalization is based on initial training period, during which a known data sequence is sent to identify channel coefficients. When the training is completed, the equalizer enters its decision-directed mode, aiming at retrieving the information symbols. Due to severe time variations in channel characteristic, as it is the case in a mobile wireless HF communication system, the training sequence has to be sent periodically to update the estimate, thereby reducing the effective channel rate. In addition, time-varying multipath propagation can cause significant channel fading, leading to system outage and equalizer failure during the training periods. It is desirable that the channel be equalized without using training signal, that is, in a blind manner, by using only the received signal.

The first blind channel equalization methods were based on a single-input single-output (SISO) channel models, sam- pled at the symbol rate. Some of them, such as the constant modulus algorithms (CMAs), involve nonlinear optimization and higher-order statistics (cummulants) of the channel output $[1,2]$. An exhaustive list of references of CMA methods is given in [3]. Interesting results regarding steady-state performance analysis of CMA are presented in $[4,5]$. Accurate estimation of cummulants requires large sample sizes. Although nonminimum-phase SISO channel is invertible by an infinitely long equalizer, this equalizer is not implementable with a causal IIR filter, thus making perfect equalization an impossible objective. Tong et al. [6] analyzed the single-input multiple-output (SIMO) FIR channel model, obtained by antenna array and/or fractional output sampling (oversampling). They have shown that the multiple channels can be identified up to a scalar constant based on the second-order statistics only. Moreover, in the absence of receiver noise, SIMO FIR channels can be perfectly equalized even in the case of nonminimumphase systems. Generally, this cannot be achieved with the symbol-spaced causal equalizer. Since [6], a large body of 
work has been exploiting SIMO channel model $[7,8,9$, $10,11,12,13,14,15]$. For a comprehensive list of important contributions in this area up to 1998 , we refer to [16].

As pointed out in [11], FIR approximation of a communication channel often requires a large number of filter parameters, and the order of the filter increases with the increase of the sampling rate. It is well known that IIR filters can capture the system dynamics with fewer parameters as compared with FIR filters. In [17], it is discussed that physical microwave radio channels often exhibit long tails of weak leading and trailing terms in its impulse response. In the case of FIR filters, this creates channel undermodeling effects and degradation of equalizer performance. IIR channel representation can reduce the effect of modeling errors.

In this paper, we propose an adaptive (self-tuning) equalizer performing sequential data processing, making it candidate for online implementations. For simplicity of presentation, single-input two-output channel model is considered. The paper is organized as follows. Section 2 describes problem statement and proposed equalizer. Section 3 presents convergence properties of developed estimators. It is shown that the parameter estimates of the unknown channel coefficients converge (a.s.) toward the scalar multiple of true parameters, while the equalizer output converges to a scalar version of the actual symbol sequence. Simulation example confirming theoretical results is presented in Section 4.

\section{PROBLEM STATEMENT AND THE BLIND EQUALIZATION ALGORITHM}

The standard model of fractionally spaced receiver is SIMO system. For the simplicity of our presentation, we consider the single-input two-output system model, or $T / 2$ fractionally spaced equalizer, where $T$ denotes the baud or symbol duration. As shown in Figure 1, in this case, the receiver performs two measurements, $x_{1}(i)$ and $x_{2}(i)$, for each transmitted symbol $w(i)$, where $i=0,1,2, \ldots$, is discrete time. Here $z^{-1}$ is a unit delay time, integer $d$ is a delay between the input $w(i)$ and the outputs $x_{k}(i), k=1,2$, while $B_{1}\left(z^{-1}\right) / A_{1}\left(z^{-1}\right)$ and $B_{2}\left(z^{-1}\right) / A_{2}\left(z^{-1}\right)$ are stable IIR transfer operators. An equivalent representation of this process is given in Figure 2, where

$$
\begin{aligned}
& A\left(z^{-1}\right)=1+a_{1} z^{-1}+\cdots+a_{n_{A}} z^{-n_{A}} \\
& B\left(z^{-1}\right)=b_{0}+b_{1} z^{-1}+\cdots+b_{L} z^{-L} \\
& C\left(z^{-1}\right)=c_{0}+c_{1} z^{-1}+\cdots+c_{L} z^{-L} .
\end{aligned}
$$

Since our channel model assumes that the delay between $w(i)$ and $x_{k}(i), k=1,2$, is equal to $d$ samples, at least one of the coefficients $b_{0}$ or $c_{0}$ in (1) must be different than zero. Otherwise, the above delay will not be equal $d$, but $d+1$ samples. For the purpose of our analysis, we assume that $c_{0} \neq 0$.

Obviously, $B=B_{1} A_{2}, C=B_{2} A_{1}$, and $A=A_{1} A_{2}$. In (1), $L=\max \left(\operatorname{deg} B\left(z^{-1}\right), \operatorname{deg} C\left(z^{-1}\right)\right)$. Assuming that there is no

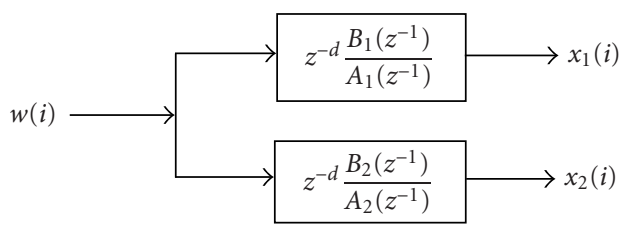

Figure 1: Channel model.

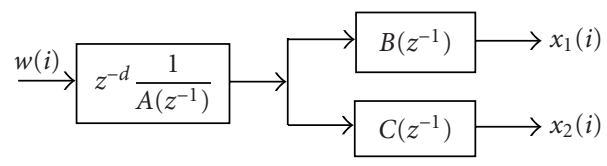

Figure 2: Equivalent channel model.

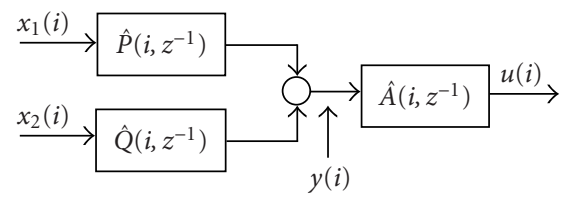

Figure 3: Channel equalizer.

receiver noise, the received signals $x_{1}(i)$ and $x_{2}(i)$ are given by

$$
\begin{aligned}
& A\left(z^{-1}\right) x_{1}(i)=z^{-d} B\left(z^{-1}\right) w(i), \\
& A\left(z^{-1}\right) x_{2}(i)=z^{-d} C\left(z^{-1}\right) w(i),
\end{aligned}
$$

for all $i \geq 0$. The signals $w(i), x_{1}(i), x_{2}(i)$ and the coefficients of $A\left(z^{-1}\right), B\left(z^{-1}\right)$, and $C\left(z^{-1}\right)$ can be complex quantities.

Our objective is online blind channel identification and equalization, that is, estimation, up to a scaling constant, of unknown polynomials $A\left(z^{-1}\right), B\left(z^{-1}\right)$, and $C\left(z^{-1}\right)$, and information symbol $w(i)$, based only on the received signals $x_{1}(i)$ and $x_{2}(i), i \geq 0$.

The proposed equalizer is depicted in Figure 3 where

$$
\begin{aligned}
& \hat{A}\left(i, z^{-1}\right)=1+\hat{a}_{1}(i) z^{-1}+\cdots+\hat{a}_{N_{A}}(i) z^{-n_{A}}, \\
& \hat{P}\left(i, z^{-1}\right)=\hat{p}_{0}(i)+\hat{p}_{1}(i) z^{-1}+\cdots+\hat{p}_{M}(i) z^{-M}, \\
& \hat{Q}\left(i, z^{-1}\right)=\hat{q}_{0}(i)+\hat{q}_{1}(i) z^{-1}+\cdots+\hat{q}_{M}(i) z^{-M},
\end{aligned}
$$

with $M=L-1$, where $\hat{A}\left(i, z^{-1}\right)$ is an estimate of $A\left(z^{-1}\right)$, recursively generated at each time instant $i$, while $\hat{P}\left(i, z^{-1}\right)$ and $\hat{Q}\left(i, z^{-1}\right)$ are obtained from the following Bezout identity:

$$
\hat{P}\left(i, z^{-1}\right) \cdot \hat{B}\left(i, z^{-1}\right)+\hat{Q}\left(i, z^{-1}\right) \cdot \hat{C}\left(i, z^{-1}\right)=1
$$

for all $i \geq 0$. Here, $\hat{B}\left(i, z^{-1}\right)$ and $\hat{C}\left(i, z^{-1}\right)$ are estimates of 
$B\left(z^{-1}\right)$ and $C\left(z^{-1}\right)$, respectively, and are given by

$$
\begin{aligned}
& \hat{B}\left(i, z^{-1}\right)=\hat{b}_{0}(i)+\hat{b}_{1}(i) z^{-1}+\cdots+\hat{b}_{L}(i) z^{-L}, \\
& \hat{C}\left(i, z^{-1}\right)=\hat{c}_{0}(i)+\hat{c}_{1}(i) z^{-1}+\cdots+\hat{c}_{L}(i) z^{-L} .
\end{aligned}
$$

We now propose two recursive algorithms providing the convergence of $\hat{B}, \hat{C}$, and $\hat{A}$ toward the scalar multiple of unknown polynomials $B, C$, and $A$. We then show that in the limit, the equalizer output $u(i)$ approaches the scalar version of the unknown symbol $w(i)$. Since $C\left(z^{-1}\right) x_{1}(i)=$ $B\left(z^{-1}\right) x_{2}(i)$, we can write

$$
x_{1}(i)=\varphi(i)^{\dagger} \theta^{*} \text {, }
$$

where $†$ stands for conjugate transpose while

$$
\begin{aligned}
\theta^{*}= & {\left[\frac{c_{1}}{c_{0}}, \ldots, \frac{c_{L}}{c_{0}} ; \frac{b_{0}}{c_{0}}, \frac{b_{1}}{c_{0}}, \ldots, \frac{b_{L}}{c_{0}}\right]^{T}, } \\
\varphi(i)^{T}= & {\left[-x_{1}(i-1), \ldots,-x_{1}(i-L) ;\right.} \\
& \left.x_{2}(i), x_{2}(i-1), \ldots, x_{2}(i-L)\right],
\end{aligned}
$$

with $(\cdot)^{T}$ being the usual transpose operation. In $(7), c_{0}$ is the leading coefficient of $C\left(z^{-1}\right)$.

Assuming that the order $L$ is known, we can use the following weighted recursive least-squares algorithm to estimate $\theta^{*}$ :

$$
\begin{aligned}
\hat{\theta}(i) & =\hat{\theta}(i-1)+p(i) \varphi(i) \bar{e}(i), \\
e(i) & =x_{1}(i)-\hat{\theta}(i-1)^{\dagger} \varphi(i), \\
p(i) & =\frac{p(i-1)}{\lambda}-\frac{(p(i-1) / \lambda) \varphi(i) \varphi(i)^{\dagger}(p(i-1) / \lambda)}{1+\varphi(i)^{\dagger}(p(i-1) / \lambda) \varphi(i)}, \\
p(0) & =p_{0} I, \quad p_{0}>0,0<\lambda<1,
\end{aligned}
$$

where in $(9) \overline{(\cdot)}$ stands for complex conjugate. In the sequel, we show that under certain conditions, $\lim _{i \rightarrow \infty} \hat{\theta}(i)=\theta^{*}$ and $\lim _{i \rightarrow \infty}\left(y(i)-z^{-d}\left(1 / A\left(z^{-1}\right)\right) w(i)\right)=0$, where (see Figure 3 )

$$
y(i)=\hat{P}\left(i, z^{-1}\right) x_{1}(i)+\hat{Q}\left(i, z^{-1}\right) x_{2}(i)
$$

with $\hat{P}\left(i, z^{-1}\right)$ and $\hat{Q}\left(i, z^{-1}\right)$ defined in (3) and (4). This motivates our algorithm for online identification of polynomial $A\left(z^{-1}\right)$. Let

$$
\begin{gathered}
\phi(i-1)^{T}=\left[-y(i-1), \ldots,-y\left(i-n_{A}\right)\right], \\
\alpha^{*}=\left[a_{1}, \ldots, a_{n_{A}}\right]^{T} .
\end{gathered}
$$

Then $\alpha^{*}$ can be estimated by using the following RLS algorithm:

$$
\begin{aligned}
\hat{\alpha}(i) & =\hat{\alpha}(i-1)+R(i) \phi(i-1) \bar{\varepsilon}(i), \\
\mathcal{E}(i) & =y(i)-\phi(i-1)^{\dagger} \hat{\alpha}(i-1), \\
R(i) & =R(i-1)-\frac{R(i-1) \phi(i-1) \phi(i-1)^{\dagger} R(i-1)}{1+\phi(i-1)^{\dagger} R(i-1) \phi(i-1)}, \\
R(0) & =r_{0} I, \quad r_{0}>0 .
\end{aligned}
$$

Note that the first stage algorithm given by (7), (8), (9), (10), and (11) is reminiscent of the concept in [7], which also uses the basic equation $C\left(z^{-1}\right) x_{1}(i)=B\left(z^{-1}\right) x_{2}(i)$. The main difference is that our work presents recursive algorithms and assumes IIR channel model.

\section{GLOBAL CONVERGENCE OF ADAPTIVE EQUALIZER}

In order to simplify algebraic details of our analysis, we consider the case where all variables are real valued. The obtained results can easily be extended to the case of complex variables. The following is assumed throughout the sequel.

Assumption 1. (i) Operators $A\left(z^{-1}\right)$ is a stable polynomial.

(ii) Polynomials $B\left(z^{-1}\right)$ and $C\left(z^{-1}\right)$ have no common factors.

Assumption 2. Signal $\{w(i)\}$ is a zero-mean sequence of mutually independent random variables satisfying $\sup _{i}|w(i)| \leq$ $k_{w}<\infty$, and

$$
\lim _{n \rightarrow 0} \frac{1}{n} \sum_{i=1}^{n} w(i)^{2}=\sigma_{w}^{2} \quad \text { (a.s.). }
$$

Assumptions 1 and 2 are standard conditions in the literature on second-order approaches for blind identification/equalization of SIMO channels. We note that [18] discusses a class of channels that do not satisfy Assumption 1(ii).

Let $F_{i}$ be an increasing sequence of $\sigma$-algebras generated by $\{w(0), w(1), \ldots, w(i)\}$. Then $\{w(i)\}$ satisfying Assumption 2 is a martingale difference sequence with respect to $F_{i}$, that is, $w(i)$ is $F_{i}$ measurable and $E\left\{w(i) \mid F_{i-1}\right\}=$ 0 (a.s.) for all $i$. For future reference, we give the convergence theorem for martingale difference sequencies.

Lemma 1 (Stout [19]). Let $w(i)$ be martingale difference sequence with respect to $F_{i}$ and let $f(i-1)$ be an $F_{i-1}$ measurable sequence. Then

$$
\left|\sum_{i=1}^{n} f(i-1) w(i)\right|=o\left(\sum_{i=1}^{n} f(i-1)^{2}\right)+O(1) \quad \text { (a.s.) }
$$


Theorem 1. Let Assumptions 1 and 2 hold, and order $L$ is known. Then algorithm (9), (10), and (11) provides

$$
\begin{aligned}
& \lim _{i \rightarrow \infty} \hat{B}\left(i, z^{-1}\right)=\frac{1}{c_{0}} B\left(z^{-1}\right) \quad \text { (a.s.), } \\
& \left.\lim _{i \rightarrow \infty} \hat{C}\left(i, z^{-1}\right)=\frac{1}{c_{0}} C\left(z^{-1}\right) \quad \text { (a.s. }\right),
\end{aligned}
$$

where $B, C$, and $\hat{B}, \hat{C}$ are defined in (1) and (5), respectively.

Proof. Let

$$
\tilde{\theta}(i)=\hat{\theta}(i)-\theta^{*},
$$

where $\theta^{*}$ is given by (7). Then, from (6) and (10), we have

$$
e(i)=-\varphi(i)^{T} \tilde{\theta}(i-1)
$$

Substituting (24) in (9) gives

$$
p(i)^{-1} \tilde{\theta}(i)=p(i)^{-1} \tilde{\theta}(i-1)-\varphi(i) \varphi(i)^{T} \tilde{\theta}(i-1)
$$

Since (11) implies

$$
p(i)^{-1}=\lambda p(i-1)^{-1}+\varphi(i) \varphi(i)^{T}
$$

equation (25) yields $p(i)^{-1} \tilde{\theta}(i)=\lambda p(i-1)^{-1} \tilde{\theta}(i-1)$, wherefrom it follows that

$$
p(i)^{-1} \tilde{\theta}(i)=\lambda^{i} p(0)^{-1} \tilde{\theta}(0)
$$

We now show that $p(i)^{-1}$ is a positive definite matrix. It is well known that Assumptions 1 and 2 imply (see [20, 21])

$$
\lim _{N \rightarrow \infty} \inf \frac{1}{N} \sum_{k=t}^{t+N} \varphi(k) \varphi(k)^{T} \geq \sigma^{*} I, \quad \sigma^{*}>0 \text { (a.s.), }
$$

for all $t \geq 0$.

Let $M_{1}$ and $M_{2}$ be quadratic matrices. Then, in the above notation, the statement $M_{1} \geq M_{2}$ implies that $x^{T} M_{1} x \geq$ $x^{T} M_{2} x$ for all nonzero vectors $x$. From (28), we conclude that there exists finite $N_{0}$ such that

$$
\frac{1}{N_{0}} \sum_{k=t}^{t+N_{0}} \varphi(k) \varphi(k)^{T} \geq \frac{\sigma^{*}}{2} I, \quad \forall t \geq 0 \text { (a.s.) }
$$

which is equivalent to

$$
\sum_{k=i-N_{0}}^{i} \varphi(i) \varphi(i)^{T} \geq \varepsilon_{1}^{*} I, \quad \forall i(\text { a.s. })
$$

where $\varepsilon_{1}^{*}=\left(\sigma^{*} / 2\right) N_{0}$. Relations (26) and (30) imply that

$$
\begin{aligned}
p(i)^{-1} & =\lambda^{i} p(0)^{-1}+\sum_{k=1}^{i} \lambda^{i-k} \varphi(k) \varphi(k)^{T} \\
& \geq \sum_{k=i-N_{0}}^{i} \lambda^{i-k} \varphi(k) \varphi(k)^{T} \\
& \geq \lambda^{N_{0}} \sum_{k=i-N_{0}}^{i} \varphi(k) \varphi(k)^{T} \geq \varepsilon_{2}^{*} I \quad \text { (a.s.) }
\end{aligned}
$$

for all $i$ and $\varepsilon_{2}^{*}=\lambda_{1}^{-N_{0}} \varepsilon_{1}^{*}$. By using (31) in (27), we can derive

$$
\|\tilde{\theta}(i)\| \leq \lambda^{i} \frac{1}{\varepsilon_{2}^{*}}\left\|p(0)^{-1} \tilde{\theta}(0)\right\| \quad \text { (a.s.) }
$$

from where the statement of the theorem directly follows.

Note that $\theta^{*}$ in (7) can become very large if $c_{0}$ is too small, which may create numerical problems when algorithm (9) attempts to estimate $\theta^{*}$. This problem can be avoided by using another parameter instead of $c_{0}$. We can define $\theta^{*}$ as follows:

$$
\theta^{*}=\left[\frac{c_{0}}{c_{1}}, \frac{c_{2}}{c_{1}}, \frac{c_{3}}{c_{1}}, \ldots, \frac{c_{L}}{c_{1}}, \frac{b_{0}}{c_{1}}, \ldots, \frac{b_{L}}{c_{1}}\right]
$$

assuming that $c_{1}$ is not close to zero. Then we can write

$$
x_{1}(i-1)=\varphi(i)^{\dagger} \theta^{*}
$$

with

$$
\begin{aligned}
\varphi(i)^{T}=[ & -x_{1}(i),-x_{1}(i-2),-x_{1}(i-3), \ldots, \\
& \left.-x_{1}(i-L), x_{2}(i), \ldots, x_{2}(i-L)\right] .
\end{aligned}
$$

As before, $\theta^{*}$ can be estimated by using algorithm (9), where $e(i)=x_{1}(i-1)-\hat{\theta}(i-1)^{\dagger} \varphi(i)$. Instead of $c_{1}$, we can similarly use other parameters, including $b_{0}, b_{1}, \ldots, b_{L}$. Except for a few minor algebraic steps, convergence analysis stays the same as before.

\subsection{Estimation of polynomial $A\left(z^{-1}\right)$}

Note that, from Figures 2 and 3,

$$
y(i)=\left(\hat{P}\left(i, z^{-1}\right) \frac{B\left(z^{-1}\right)}{c_{0}}+\hat{Q}\left(i, z^{-1}\right) \frac{C\left(z^{-1}\right)}{c_{0}}\right)\left(c_{0} z(i)\right),
$$

where

$$
z(i)=z^{-d} \frac{1}{A\left(z^{-1}\right)} w(i)
$$


Equation (36) can be written in the form

$$
\begin{aligned}
y(i)=( & \hat{P}\left(i, z^{-1}\right) \cdot \hat{B}\left(i, z^{-1}\right) \\
& \left.+\hat{Q}\left(i, z^{-1}\right) \cdot \hat{C}\left(i, z^{-1}\right)\right)\left(c_{0} z(i)\right)+\delta(i)
\end{aligned}
$$

with

$$
\begin{aligned}
\delta(i)=\left[\hat{P}\left(i, z^{-1}\right) \cdot\left(\frac{B\left(z^{-1}\right)}{c_{0}}-\hat{B}\left(i, z^{-1}\right)\right)\right. \\
\left.\quad+\hat{Q}\left(i, z^{-1}\right) \cdot\left(\frac{C\left(z^{-1}\right)}{c_{0}}-\hat{C}\left(i, z^{-1}\right)\right)\right]\left(c_{0} z(i)\right) .
\end{aligned}
$$

Since symbols $w(i)$ are uniformly bounded and $A\left(z^{-1}\right)$ is a stable operator, $z(i)$ is bounded for all $i \geq 0$. Hence, from (22), one obtains

$$
\lim _{i \rightarrow \infty} \delta(i)=0 \quad \text { (a.s.). }
$$

We now turn attention to Bezout identity (4). Let

$$
H=\left[\begin{array}{cccccccc}
b_{0} & 0 & \cdots & 0 & c_{0} & 0 & \cdots & 0 \\
b_{1} & b_{0} & & 0 & c_{1} & c_{0} & & 0 \\
b_{2} & b_{L} & & 0 & c_{2} & c_{1} & & 0 \\
\vdots & \vdots & & \vdots & \vdots & \vdots & & \vdots \\
b_{L-1} & b_{L-2} & \cdots & b_{0} & c_{L-1} & c_{L-2} & & c_{0} \\
b_{L} & b_{L-1} & \cdots & b_{1} & c_{L} & c_{L-1} & & c_{1} \\
0 & b_{L} & \cdots & b_{2} & 0 & c_{L} & & c_{2} \\
\vdots & \vdots & & \vdots & \vdots & \vdots & & \vdots \\
0 & 0 & \cdots & b_{L-1} & 0 & 0 & & c_{L-1} \\
0 & 0 & \cdots & b_{L} & 0 & 0 & \cdots & c_{L}
\end{array}\right]
$$

be $2 L \times 2 L$ eliminant (Sylvester resultant) matrix of polynomials $B\left(z^{-1}\right)$ and $C\left(z^{-1}\right)$. Assumption 1 implies that for some $\varepsilon_{3}^{*}>0$,

$$
|\operatorname{det}(H)| \geq \varepsilon_{3}^{*}>0,
$$

where $\operatorname{det}(H)$ denotes determinant of $H$. Let $\hat{H}(i)$ be eliminant matrix of polynomials $\hat{B}\left(i, z^{-1}\right)$ and $\hat{C}\left(i, z^{-1}\right)$. Then, by (42) and Theorem 1 , there exists some $i_{0}$ such that

$$
|\operatorname{det}(H)| \geq \varepsilon_{4}^{*}>0 \quad \text { (a.s.) }
$$

for all $i \geq i_{0}$, and some $\varepsilon_{4}^{*}$. Hence, (4) has a solution for all $i \geq i_{0}$. Then, from (4) and (38), it follows that

$$
y(i)=c_{0} z(i)+\delta(i) \quad(\text { a.s. })
$$

for all $i \geq i_{0}$. Substituting (37) into (44), one obtains

$$
A\left(z^{-1}\right) y(i)=c_{0} w(i-d)+A\left(z^{-1}\right) \delta(i) \quad(\text { a.s. })
$$

or

$$
y(i)=\phi(i-1)^{T} \alpha^{*}+c_{0} w(i-d)+A\left(z^{-1}\right) \delta(i) \quad(\text { a.s. })
$$

with $\phi(i)$ and $\alpha^{*}$ defined in (14) and (15). The parameter vector $\alpha^{*}$ is estimated by using algorithm (16), (17), and (18). Next, we show the consistency of the parameter estimates $\hat{\alpha}(i)$.

Theorem 2. Let Assumptions 1 and 2 hold. Then $\hat{\alpha}(i)$ generated by the algorithm (16), (17), and (18) satisfies

$$
\lim _{i \rightarrow \infty} \hat{\alpha}(i)=\alpha^{*} \quad(\text { a.s. }) .
$$

Proof. First we show that the regressor $\phi(i)$ is persistently exciting $(\mathrm{PE})$, that is,

$$
\liminf _{i \rightarrow \infty} \frac{1}{i} \sum_{k=1}^{i} \phi(k) \phi(k)^{T} \geq \varepsilon_{5}^{*} I, \quad \varepsilon_{5}^{*}>0 \text { (a.s.). }
$$

Note that by using (44), $\phi(i)$ given by (14) can be written in the form

$$
\phi(k)=\varsigma(k)+\psi(k)
$$

where

$$
\begin{aligned}
\varsigma(k)^{T} & =\left[-c_{0} z(k-1), \ldots,-c_{0} z\left(k-n_{A}\right)\right], \\
\psi(k)^{T} & =\left[-\delta(k-1), \ldots,-\delta\left(k-n_{A}\right)\right] .
\end{aligned}
$$

Since $A\left(z^{-1}\right)$ is a stable operator, Assumption 2 implies (see $[20,21])$

$$
\liminf _{i \rightarrow \infty} \frac{1}{i} \sum_{k=1}^{i} \varsigma(k) \varsigma(k)^{T} \geq \varepsilon_{5}^{*} I \quad \text { (a.s.) }
$$

for some $\varepsilon_{5}^{*}>0$. Using the fact that $z(i)$ is finite sequence, and by $(40) \psi(k) \underset{k \rightarrow \infty}{\longrightarrow} 0$ (a.s.), application of the CauchySchwartz's inequality yields

$$
\begin{aligned}
\left\|\frac{1}{i} \sum_{k=1}^{i} \varsigma(k) \psi(k)^{T}\right\| \leq & \left(\frac{1}{i} \sum_{k=1}^{i}\|\varsigma(k)\|^{2}\right)^{1 / 2} \\
& \cdot\left(\frac{1}{i} \sum_{k=1}^{i}\|\psi(k)\|^{2}\right)^{1 / 2} \underset{i \rightarrow \infty}{\longrightarrow} 0 \quad \text { (a.s.). }
\end{aligned}
$$


Also from (49), we obtain

$$
\begin{aligned}
\phi(k) \phi(k)^{T}= & \varsigma(k) \varsigma(k)^{T}+\varsigma(k) \psi(k)^{T} \\
& +\psi(k) \varsigma(k)^{T}+\psi(k) \psi(k)^{T} .
\end{aligned}
$$

Then statement (48) follows from (52), (53), and (54). We now analyze recursion (16). Observe that from (46), we can derive

$$
\begin{aligned}
y(i)-\phi(i-1)^{T} \hat{\alpha}(i-1)= & -\phi(i-1)^{T} \tilde{\alpha}(i-1) \\
& +c_{0} w(i-d)+A\left(z^{-1}\right) \delta(i),
\end{aligned}
$$

where

$$
\tilde{\alpha}(i)=\hat{\alpha}(i)-\alpha^{*} .
$$

Hence from (16), it follows that

$$
\begin{aligned}
R(i)^{-1} \tilde{\alpha}(i)= & R(i)^{-1} \tilde{\alpha}(i-1) \\
& +\phi(i-1)\left[-\phi(i-1)^{T} \tilde{\alpha}(i)+c_{0} w(i-d)\right. \\
& \left.+A\left(z^{-1}\right) \delta(i)\right] .
\end{aligned}
$$

Since

$$
R(i)^{-1}=R(i-1)^{-1}+\phi(i-1) \phi(i-1)^{T},
$$

the previous equation gives

$$
\begin{aligned}
R(i)^{-1} \tilde{\alpha}(i)= & R(i-1)^{-1} \tilde{\alpha}(i-1) \\
& +\phi(i-1)\left[c_{0} w(i-d)+A\left(z^{-1}\right) \delta(i)\right],
\end{aligned}
$$

from where we have

$$
\begin{aligned}
R(i)^{-1} \tilde{\alpha}(i)= & R(0)^{-1} \tilde{\alpha}(0) \\
& +\sum_{k=1}^{i} \phi(k-1)\left[c_{0} w(k-d)+A\left(z^{-1}\right) \delta(k)\right] .
\end{aligned}
$$

Observe now that (4), (13) and, Theorem 1 imply bounderies of $y(i)$. Then, by $(14),\{\phi(i)\}$ is a bounded sequence. Further from (4), (9), (10), and (13), we can conclude that $y(i-1)$ depends only on the past samples $w(i-d-k), k \geq 1$, and not on $w(i-d)$. Then (14) implies that $\phi(i-1)$ is $F_{i-d-1}$ measurable. Hence, application of Lemma 1 gives

$$
\lim _{i \rightarrow \infty} \frac{1}{i} \sum_{k=0}^{i} \phi(k-1) w(k-d)=0 \quad \text { (a.s.). }
$$

Statement (61) is also intuitively clear from the fact that $\phi(k-$ $1)$ and $w(k-d)$ are bounded and independant signals, and $w(k-d)$ is a zero-mean variable. Since $\delta(k) \underset{k \rightarrow \infty}{\longrightarrow} 0$, we also
have

$$
\lim _{i \rightarrow \infty} \frac{1}{i} \sum_{k=0}^{i} \phi(k-1)\left(A\left(z^{-1}\right) \delta(k)\right)=0 \quad \text { (a.s.). }
$$

Note that (58) implies

$$
R(i)^{-1}=R(0)^{-1}+\sum_{k=0}^{i} \phi(k-1) \phi(k-1)^{T} .
$$

Then statement (47) directly follows from (48), (60), (61), and (62). This completes the proof.

Next we show that

$$
\left.\lim _{i \rightarrow \infty}\left(u(i)-c_{0} w(i-d)\right)=0 \quad \text { (a.s. }\right)
$$

where (see Figure 3)

$$
u(i)=\hat{A}\left(i, z^{-1}\right) y(i)
$$

while $c_{0}$ is an unknown leading coefficient in $C\left(z^{-1}\right)$. Note that

$$
u(i)=\left(\hat{A}\left(i, z^{-1}\right)-A\left(z^{-1}\right)\right) y(i)+A\left(z^{-1}\right) y(i) .
$$

Since, by Theorem $1, \lim _{i \rightarrow \infty}\left(\hat{A}\left(i, z^{-1}\right)-A\left(z^{-1}\right)\right)=0$ (a.s.) and $y(i)$ is bounded, (40), (45), and (66) imply statement (64).

Note that algorithm (14), (15), (16), (17), and (18) is a simple adaptive FIR linear predictor [22], and the proof of convergence in Theorem 1 takes into account the dynamics of the input to the predictor, which is the output of the first adaptive filtering stage.

\section{SIMULATION EXAMPLE}

In this experiment, we are using an i.i.d symbol sequence drawn from a 16-QAM constellation. The corresponding symbol levels along both axes are $-2,-1,1$, and 2 . The polynomials $B\left(z^{-1}\right)$ and $C\left(z^{-1}\right)$ are obtained by oversampling the following continuous time channel [9]:

$$
\begin{aligned}
h_{c}(t)= & e^{-j 2 \pi(0.15)} r_{c}(t-0.25 T, \beta) \\
& +08 e^{-j 2 \pi(0.6)} r_{c}(t-T, \beta), \quad t \in[0,4 T),
\end{aligned}
$$

where $r_{c}(t, \beta)$ is the raised cosine with roll-off factor $\beta$ while $T$ is the symbol duration. As in [9], we take $\beta=0.35$.

The above $h_{c}(t)$ represents a causal approximation of a two-ray multipath mobile radio environment. By sampling $h_{c}(t)$ at a rate of $T / 2$, we obtain

$$
\begin{aligned}
B\left(z^{-1}\right)= & (0.52-j 0.72)+(-0.48+j 0.24) z^{-1} \\
& +(-0.05+j 0.07) z^{-2}+(0.01-j 0.02) z^{-3}, \\
C\left(z^{-1}\right)= & (0.12-j 0.43)+(-0.48+j 0.41) z^{-1} \\
& +(0.13-j 0.11) z^{-2}+(-0.04+j 0.03) z^{-3} .
\end{aligned}
$$




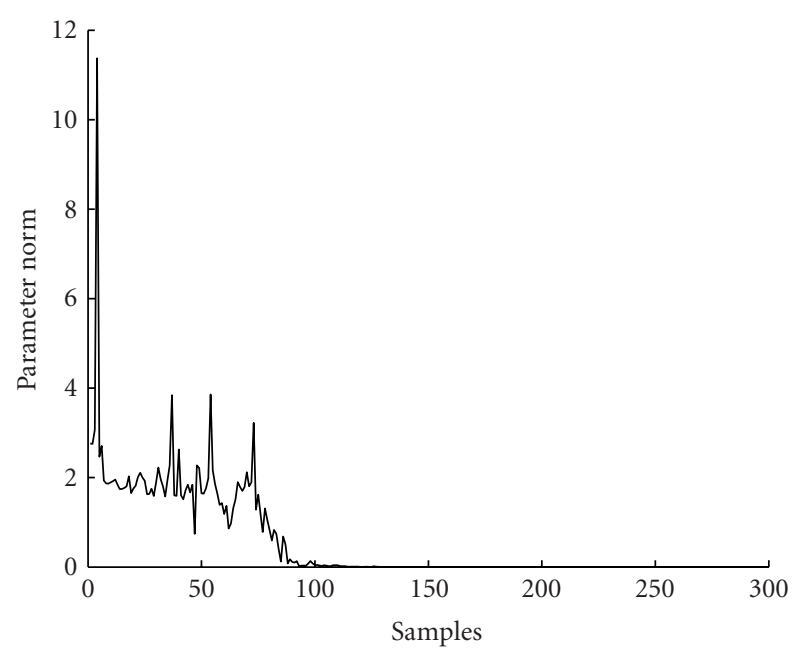

FIGURE 4: Norm of parameter error vector.

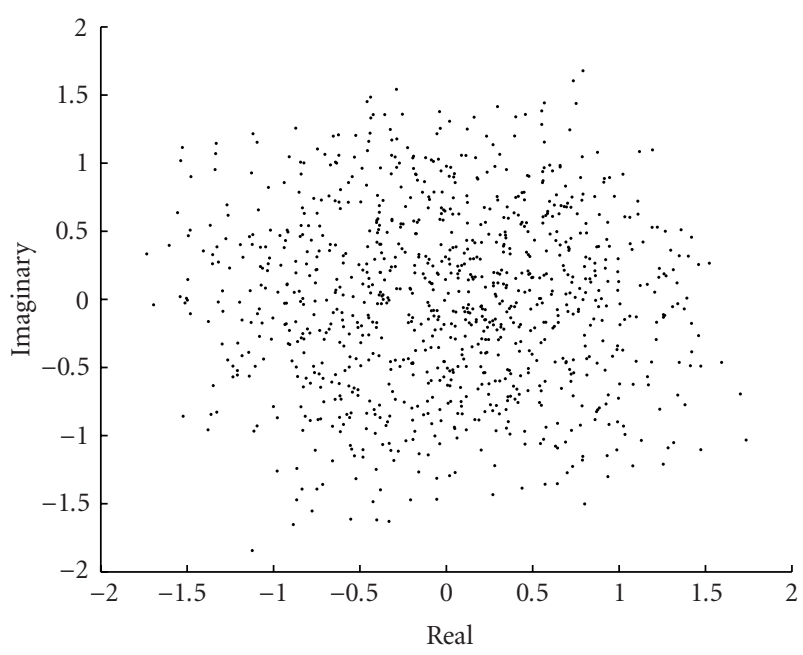

FIgURE 5: Eye diagram of channel output.

In our simulations, we assume that

$$
A\left(z^{-1}\right)=1+0.8 z^{-1}+0.41 z^{-2} .
$$

Parameter estimation error is depicted in Figure 4. Figure 5 presents received symbols while Figure 6 shows the equalized symbol-eye diagram. The amount of rotation and magnification in the eye diagram is a function of $c_{0}=(0.12-j 0.43)$, that is, angle of rotation is $-73.86^{\circ}$, while the magnification is $\left|c_{0}\right|=0.45$. Figure 7 shows the following minimum mean square error on a sample path over 1000 symbols:

$$
J(n)=\frac{1}{2} \sum_{i=1}^{n}\left(u(i)-c_{0} w(i-d)\right)^{2},
$$

where $c_{0}$ is the leading coefficient in $C\left(z^{-1}\right)$. Obviously, $J(n)$

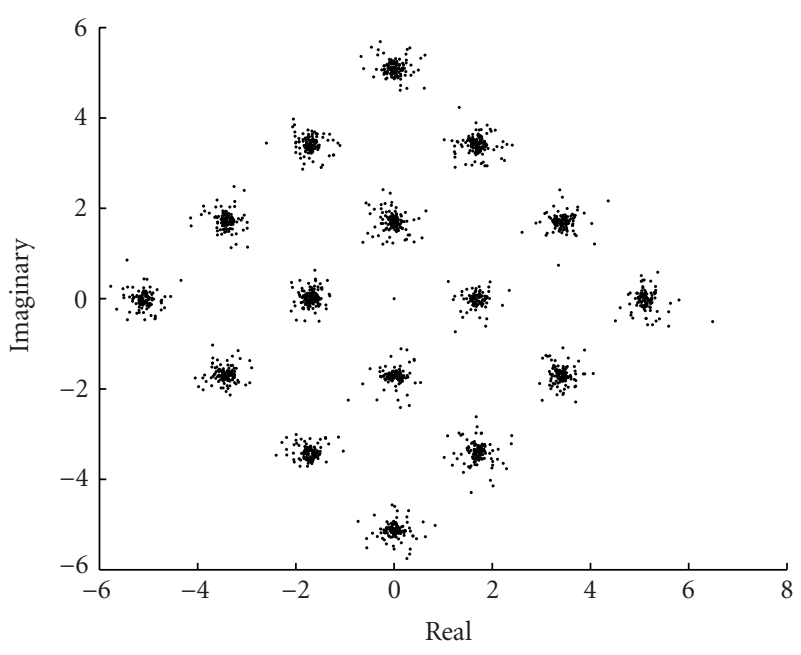

FIGURE 6: Eye diagram of equalizer output.

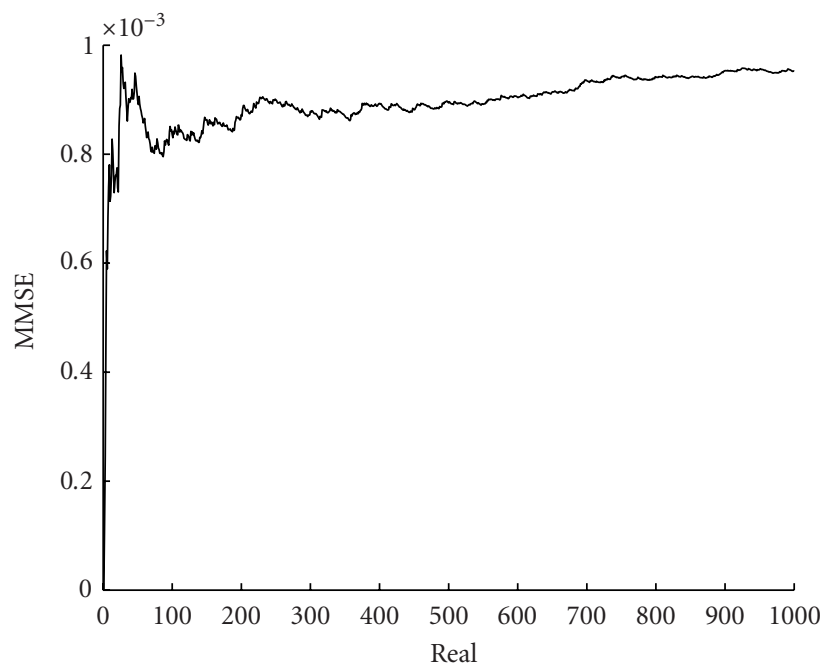

Figure 7: Minimum mean square error.

slowly approaches zero value. It is not difficult to see that all four plots coincide with the theoretical conclusions.

\section{CONCLUSION}

The self-tuning blind equalization is considered in this paper. The proposed method consists of two recursive estimators: one for estimation of "FIR portion" of the channel, while the second algorithm estimates "IIR portion" (denominator) of the channel. It is proved that the first estimator converges (a.s) toward a scalar multiple of the true parameters, and the second algorithm provides (a.s) consistent parameter estimates. Moreover, it is shown that the equalizer output converges toward the scaled version of actual symbol sequence. It is well known that the presence of receiver noise will adversely affect equalizer performance. Currently under way are efforts to extend the above results to the case 
when such noise is present, and replace RLS algorithms with LMS type procedures. The choice of the order of IIR channel model is an important design step. If this order is selected to be too small, unmodelled channel dynamics can cause deterioration in equalizer performance. Performance analysis of some second-order methods for blind identification/equalization with respect to channel undermodeling is presented in [17]. Similar analysis for our algorithms is worth further investigation.

\section{ACKNOWLEDGMENTS}

This work was supported in part by NASA Grant NAG510716 and in part by the State of Utah Center of Excellence Program.

\section{REFERENCES}

[1] D. N. Godard, "Self recovering equalization and carrier tracking in two-dimensional data communication systems," IEEE Trans. Communications, vol. 28, no. 11, pp. 1867-1875, 1980.

[2] O. Shalvi and E. Weinstein, "New criteria for blind deconvolution of nonminimum phase systems (channels)," IEEE Transactions on Information Theory, vol. 36, no. 2, pp. 312321, 1990.

[3] C. R. Johnson Jr, P. Schniter, T. J. Endres, J. D. Behm, D. R. Brown, and R. A. Casas, "Blind equalization using the constant modulus criterion: a review," Proceedings of the IEEE, vol. 86, no. 10, pp. 1927-1950, 1998.

[4] J. Mai and A. H. Sayed, "A feedback approach to the steadystate performance of fractionally spaced blind adaptive equalizers," IEEE Trans. Signal Processing, vol. 48, no. 1, pp. 80-91, 2000.

[5] P. Schniter and C. R. Johnson Jr, "Bounds for the MSE performance of constant modulus estimators," IEEE Transactions on Information Theory, vol. 46, no. 7, pp. 2544-2560, 2000.

[6] L. Tong, G. Xu, and T. Kailath, "Blind identification and equalization based on second-order statistics: a time domain approach," IEEE Transactions on Information Theory, vol. 40, no. 2, pp. 340-349, 1994.

[7] E. Moulines, P. Duhamel, J.-F. Cardoso, and S. Mayrargue, "Subspace methods for the blind identification of multichannel FIR filters," IEEE Trans. Signal Processing, vol. 43, no. 2, pp. 516-525, 1995.

[8] K. Abed-Meraim, E. Moulines, and P. Loubaton, "Prediction error method for second-order blind identification," IEEE Trans. Signal Processing, vol. 45, no. 3, pp. 694-705, 1997.

[9] Giannakis, G. B. and Halford, S. D., "Blind fractionally spaced equalization of noisy FIR channels: direct and adaptive solutions," IEEE Trans. Signal Processing, vol. 45, no. 9, pp. 22772292, 1997.

[10] G. B. Giannakis and C. Tepedelenlioglu, "Direct blind equalizers of multiple FIR channels: a deterministic approach," IEEE Trans. Signal Processing, vol. 47, no. 1, pp. 62-74, 1999.

[11] E. W. Bai and M. Fu, "Blind system identification and channel equalization of IIR systems without statistical information," IEEE Trans. Signal Processing, vol. 47, no. 7, pp. 1910-1921, 1999.

[12] A. Gorokhov and P. Loubaton, "Blind identification of MIMO-FIR systems: A generalized linear prediction approach," Signal Processing, vol. 73, no. 1-2, pp. 105-124, 1999.

[13] D. Gesbert and P. Duhamel, "Unbiased blind adaptive channel identification and equalization," IEEE Trans. Signal Processing, vol. 48 , no. 1, pp. 148-158, 2000.
[14] H. Gazzah, P. A. Regalia, J.-P. Delmas, and K. Abed-Meraim, "A blind multichannel identification algorithm robust to order overestimation," IEEE Trans. Signal Processing, vol. 50, no. 6, pp. 1449-1458, 2002.

[15] Z.-Q. Luo, M. Meng, K. M. Wong, and J.-K. Zhang, "A fractionally spaced blind equalizer based on linear programming," IEEE Trans. Signal Processing, vol. 50, no. 7, pp. 1650-1660, 2002.

[16] L. Tong and S. Perreau, "Multichannel blind identification: from subspace to maximum likelihood methods," Proceedings of the IEEE, vol. 86, no. 10, pp. 1951-1968, 1998.

[17] J.-P. Delmas, H. Gazzah, A. P. Liavas, and P. A. Regalia, "Statistical analysis of some second-order methods for blind channel identification/equalization with respect to channel undermodeling," IEEE Trans. Signal Processing, vol. 48, no. 7, pp. 1984-1998, 2000.

[18] J. K. Tugnait, "On blind identifiability of multipath channels using fractional sampling and second-order cyclostationary statistics," IEEE Transactions on Information Theory, vol. 41, no. 1, pp. 308-311, 1995.

[19] W. F. Stout, Almost Sure Convergence, Academic Press, New York, NY, USA, 1974.

[20] L. Ljung and T. Söderström, Theory and Practice of Recursive Identification, MIT Press, Cambridge, Mass, USA, 1983.

[21] G. C. Goodwin and K. S. Sin, Adaptive Filtering, Prediction and Control, Prentice Hall, Englewood Cliffs, NJ, USA, 1984.

[22] S. Haykin, Adaptive Filter Theory, Prentice Hall, Upper Saddle River, NJ, USA, 2002.

Miloje Radenkovic received the Diploma of Engineering degree in 1978, M.S. degree in 1982, and Ph.D. degree in 1986, all in electrical engineering, from Belgrade University, Yugoslavia. From 1979 to 1986, he was a Research Scientist in the Technical Institute in Belgrade. From 1986 to 1990, he was a Docent at Novi Sad University, Zrenjanin, Yugoslavia. During the academic year 1990/1991, he was a Visiting Professor at the Department of Electrical Engineering at Notre Dame University, India. He is presently an Associate Professor of electrical engineering at the University of Colorado at Denver. Dr. Radenkovic research includes adaptive systems in control, signal processing, and communications.

Tamal Bose received the Ph.D. degree in electrical engineering from Southern Illinois University in 1988. He is currently a Professor of electrical and computer engineering at Utah State University at Logan, and Director of the Center for High-speed Information Processing (CHIP). Dr. Bose served as the Associate Editor for the IEEE Transactions on Signal Processing from 1992 to 1996. He is currently on the editorial board of the IEICE Transactions on Fundamentals of Electronics, Communications and Computer Sciences, Japan. Dr. Bose received the 2002 Research Excellence Award from the College of Engineering at Utah State University. He received the Researcher of the Year and Service Person of the Year Awards from the University of Colorado at Denver and the Outstanding Achievement Award at the Citadel. He also received two Exemplary Researcher Awards from the Colorado Advanced Software Institute. $\mathrm{He}$ is a Senior Member of the IEEE.

Zhurun Zhang received the M.S. degree in electrical and computer engineering from Utah State University in 2002. From 1994 to 2000, he studied and researched in Shanghai Jiao Tong University, Shanghai, China. Mr. Zhang received the B.S. degree in electrical engineering in 1998 and M.S. degree in 2000 in computer engineering from Shanghai Jiao Tong University. He is currently a Software Design Engineer in Microsoft. 